\title{
A simple clinical assessment of breast animation deformity following direct-to-implant breast reconstruction
}

\author{
Diana Lydia Dyrberg ${ }^{1}$, Gudjon Leifur Gunnarsson², Camilla Bille ${ }^{3}$, Jens Ahm Sørensen ${ }^{3}$, \\ Jørn Bo Thomsen ${ }^{1}$ \\ ${ }^{1}$ Department of Plastic Surgery, Odense University Hospital, Odense and Lillebaelt Hospital, Vejle, Denmark; ${ }^{2}$ Department of Plastic Surgery, \\ Telemark Hospital, Skien, Norway; ${ }^{3}$ Department of Plastic Surgery, Odense University Hospital, Odense, Denmark
}

Background A high incidence of breast animation deformity (BAD) has been reported following immediate breast reconstruction with subpectorally placed implants. The aim of this study was to assess and compare the incidence of BAD in women who underwent either subpectoral or prepectoral immediate breast reconstruction. Therefore, we developed a grading tool and tested its reproducibility in a clinical setting.

Methods Video recordings of 37 women who had undergone unilateral or bilateral immediate breast reconstruction were evaluated by two consultant plastic surgeons. The degree of BAD was assessed by our grading tool, named the Nipple, Surrounding Skin, Entire Breast (NSE) grading scale, which evaluates the degree of tissue distortion in three areas of the breast. Blinded assessments were performed twice by each observer.

Results Eighteen patients were reconstructed with subpectoral implant placement and 19 with prepectoral implant placement. Using the NSE grading scale, we found a significant difference in the degree of BAD between the groups, in favor of patients who underwent prepectoral immediate breast reconstruction $(0.2$ vs. $4, P=0.000)$. Inter- and intraobserver agreement was moderate $(74 \%)$ to strong $(88 \%)$.

Conclusions The incidence and severity of BAD was significantly lower in women reconstructed with a prepectorally placed implant than in those who underwent subpectoral immediate breast reconstruction. All patients reconstructed using the subpectoral technique had some degree of BAD. The inter- and intraobserver agreements were high when using the NSE grading scale, suggesting it is an easy-to-use, reproducible scale for assessing BAD in women who undergo immediate breast reconstruction.

Keywords Mammaplasty / Breast deformity / Breast implant / Treatment outcome / Incidence
Correspondence: Jørn Bo Thomsen Department of Plastic Surgery, Odense University Hospital, Odense and Lillebaelt Hospital, Vejle, Sdr Boulevard 29, Odense 5000, Denmark Tel: $+45-51206760$

Fax: $+45-65413038$

E-mail: joern.bo.thomsen@rsyd.dk
This article was presented at the 37 th Congress of the Scandinavian Association of Plastic Surgeons on June 14, 2018, in Copenhagen, Denmark.

Received: April 9, $2019 \bullet$ Revised: August 28, $2019 \bullet$ Accepted: October 12, 2019

pISSN: 2234-6163・ elSSN: 2234-6171・https://doi.org/10.5999/aps.2019.00493・ Arch Plast Surg 2019;46:535-543

\section{INTRODUCTION}

Breast animation deformity (BAD), dynamic breast deformity, breast distortion, muscle flex deformity, and jumping breast are synonyms for a condition in which the shape of the breast changes or is distorted above an implant during contraction of

Copyright $\odot 2019$ The Korean Society of Plastic and Reconstructive Surgeons

This is an Open Access article distributed under the terms of the Creative Commons Attribution Non-Commercial License (http://creativecommons.org/

licenses/by-nc/4.0/) which permits unrestricted non-commercial use, distribution, and reproduction in any medium, provided the original work is properly cited.

www.e-aps.org 
the pectoralis major muscle (PMM) [1]. The degree of $\mathrm{BAD}$ is variable; however, $\mathrm{BAD}$ often affects patients' quality of life and the cosmetic outcomes of surgery [2-4]. The incidence of BAD has been reported to be as high as $77 \%$ following breast augmentation with subpectoral implant placement [5]. Many recent papers have described $\mathrm{BAD}$ following implant-based immediate breast reconstruction and reported no incidence or a very low incidence of $\mathrm{BAD}$; however, most such papers have neither described how the condition was assessed nor stated how they classified the different degrees of $\mathrm{BAD}[6]$.

In 2008, Spear et al. [5] developed a 4-point $\mathrm{BAD}$ grading scale for women undergoing breast augmentation. To our knowledge, the study by Spear et al. is the only study to have tested an assessment tool for $\mathrm{BAD}$ in a clinical setting; furthermore, the grading scale was developed for women who underwent breast augmentation, not for the assessment of reconstructed breasts. Overall, Spear's grading scale is based on a visual assessment of two different aspects: whether the implant is placed in a subpectoral or prepectoral location, and the degree of distortion of the breasts. However, when assessing patients' breasts after immediate breast reconstruction, we found that the main features of $\mathrm{BAD}$ were distortion of the skin and movement of the breasts. Based on these observations, we developed a tool specifically for the assessment of $\mathrm{BAD}$ following immediate breast reconstruction.

The popularity of implant-based breast reconstruction has increased over the last decade [7]. Immediate breast reconstruction can be performed at the time of mastectomy by using various pocket planes, including prepectoral, partial muscle coverage, and total muscle coverage [8]. The use of acellular dermal matrix $(\mathrm{ADM})$ and lipofilling have facilitated a shift towards prepectoral implant placement [9]. Prepectoral direct-to-implant breast reconstruction may reduce the incidence of $\mathrm{BAD}$. However, its potential disadvantages include an increased incidence of capsular contracture, secondary ptosis, bottoming out, and visible wrinkling $[10,11]$. The aims of this paper were to assess and quantify the incidence of $\mathrm{BAD}$ in women undergoing direct-to-implant breast reconstruction with either prepectoral or dual-plane subpectoral implant placement, to develop a grading scale for the simple clinical assessment of $\mathrm{BAD}$ following direct-to-implant breast reconstruction, and to test the reproducibility of the grading system in a clinical setting by assessing its inter- and intra-rater agreement.

\section{METHODS}

We conducted a retrospective cohort study of 37 women over 18 years of age who underwent unilateral or bilateral immediate breast reconstruction between November 2011 and December 2017. The study was a collaboration between three different Scandinavian departments of plastic surgery and was approved by the Regional Committee on Health Research Ethics for Southern Denmark (S-20160160).

All direct-to-implant breast reconstructions were performed following skin-sparing mastectomy and nipple-sparing mastectomy performed by the same team of one breast surgeon and one plastic surgeon in Norway. Two different reconstructive techniques were compared: a prepectoral pocket using a porcine-derived mesodermal matrix (Meso Biomatrix) to cover the implant and a dual-plane subpectoral pocket plane, also using an $\mathrm{ADM}$ as an inferior and lateral hammock (Fig. 1).

\section{Surgical technique \\ Mastectomy}

The skin was incised, and the breast gland was dissected of the PMM and the thoracic wall in the subglandular plane using monopolar cautery. The breast was then infiltrated with a solution of $1 \mathrm{~L} \mathrm{NaCl}$ with $1 \mathrm{~mL}$ of epinephrine using a blunt-tip cannula below the subcutaneous fascia. The mastectomy commenced using scissors for blunt and sharp dissection at the level of the subcutaneous fascia. The entire mastectomy specimen was removed en bloc. The cavity was then inspected and palpated to ensure that no glandular tissue remnants were left behind. The mastectomy flaps, skin quality, and skin viability were evaluated prior to reconstruction. We have described our mastectomy technique in previous publications $[9,12]$.

\section{Subpectoral breast reconstruction}

The direct-to-implant technique for subpectoral implant placement we used has been described in a recent publication [12] and illustrated in another recent publication [13]. The inferomedial insertion of the PMM was released and a biological mesh was sutured by $2-0$ absorbable sutures laterally and along the inframammary crease to create the implant pocket. The chosen size of the implant was based on the patients' wishes, skin quality, and measurements of the breast base and projection.

\section{Prepectoral breast reconstruction}

The authors' direct-to-implant prepectoral technique has been visually illustrated in a recent publication $[9,13]$. Following mastectomy, the circumference of the breast footprint was marked. One piece of biological mesh was sutured by a $2-0 \mathrm{ab}-$ sorbable suture to the lateral edge of the PMM, the inframammary crease, and medially towards the sternum to create a hammock for the implant.

Following either type of breast reconstruction, two drains 


\section{Fig. 1. Direct-to-implant breast reconstruction}

(A) Direct-to-implant breast reconstruction with subpectoral (dual-plane) implant placement. This figure illustrates a unilateral breast reconstruction where the implant was placed in a subpectoral pocket after the pectoralis major muscle was lifted from the thoracic wall with an inferomedial release. (B) Direct-to-implant breast reconstruction with prepectoral implant placement. This figure illustrates a unilateral breast reconstruction where the implant was placed in a prepectoral pocket.
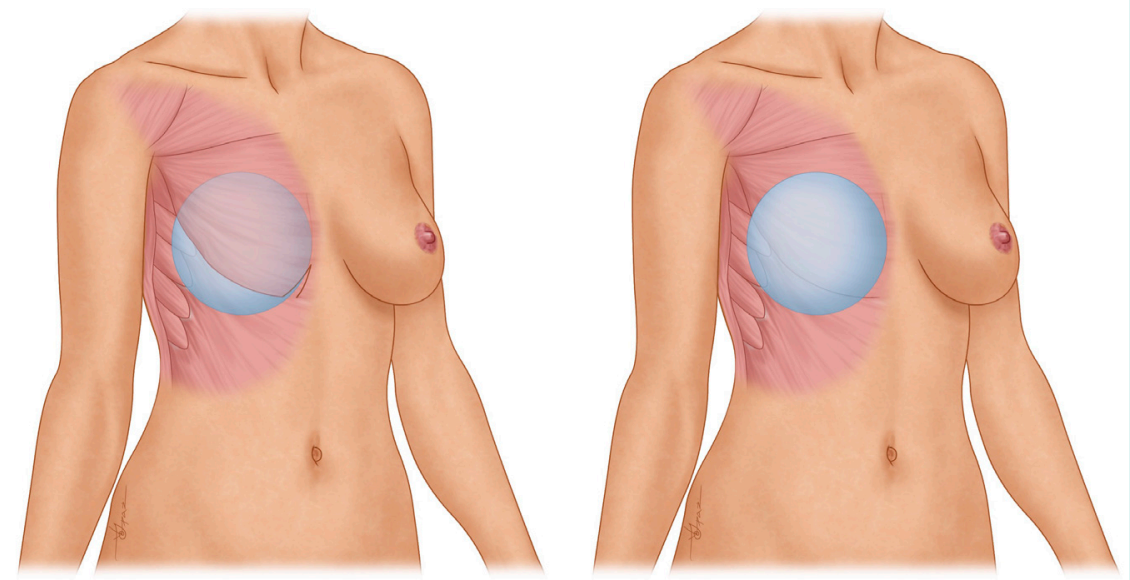

(A)

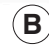

\section{Fig. 2. The NSE grading scale}

Illustrations of the three different features assessed when grading breast animation deformity using the NSE grading scale: (A) distortion of the top of the breast mound (TBM)/nipple areolar complex (NAC), (B) distortion of the breast skin surrounding the TBM/NAC, and $(\mathrm{C})$ distortion of the entire breast.

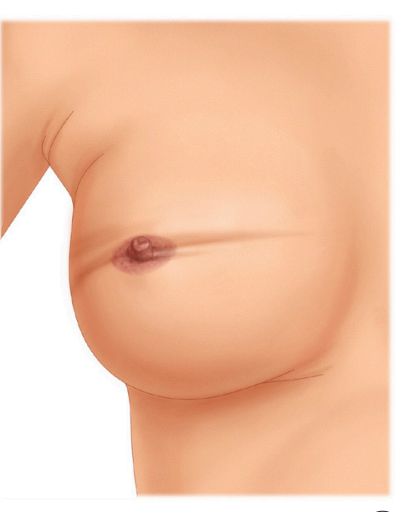

(A)

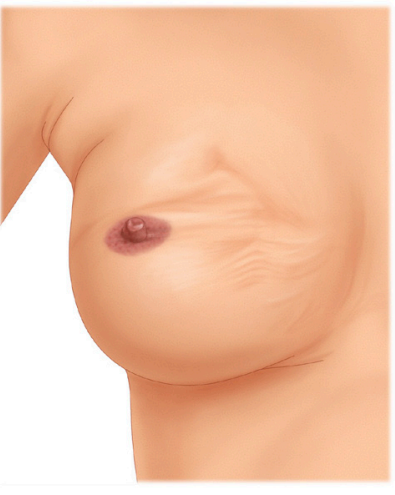

(B)

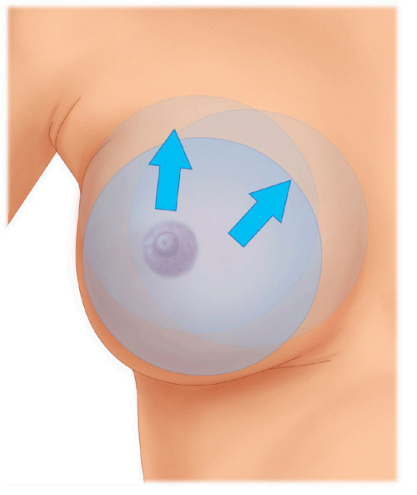

(C) were placed, one along the inframammary fold and one towards the axilla. The skin was closed in two layers using absorbable 3-0 sutures. Drains were removed postoperatively when the daily output was less than $20 \mathrm{~mL}$.

\section{Patients}

From patient records, data on demographics and information about surgical indication, prepectoral or subpectoral placement of the implant, form and size of the implant, length of stay, and number of days with the drain in place were obtained. Videos were recorded at follow-up in the outpatient clinic with the patients in the standing position, first relaxed and then performing maximal contraction of the PMM by pressing the palms of their hands together in the midline in front of the waist. The videos were recorded in Norway by the consultant plastic surgeon. The videos were labeled with numbers and randomized using an online randomizer (www.random.org) [14]. The labeled and randomized videos were then sent for assessment by two consul- tant plastic surgeons in Denmark. Both observers were blinded to all patient information during the assessment process.

\section{Grading system for the assessment of BAD following immediate breast reconstruction}

The grading system used for the assessment of $\mathrm{BAD}$ following immediate breast reconstruction focused on distortion of three aesthetic features of the breast: the top of the breast mound (TBM)/nipple areolar complex (NAC), the breast skin surrounding the TBM/NAC, and the entire breast. Therefore, we named the scale the Nipple, Surrounding Skin, Entire Breast (NSE) grading scale.

We assessed distortion and movement related to the following aesthetic features: the degree of distortion around the TBM/ $\mathrm{NAC}$, the degree of distortion of the breast skin surrounding the $\mathrm{TBM} / \mathrm{NAC}$, and the degree of movement of the entire breast (Fig. 2). As illustrated by postoperative photographs (Figs. 3-5), features 1 and 2 are distinguished by tissue differences: around 
Fig. 3. TBM/NAC distortion of grades

During activation of the pectoralis major muscle, the images show: moderate (grade 1) distortion of the breast mound (TBM)/nipple areolar complex (NAC) (A, B) and severe (grade 2) distortion of the TBM/NAC (C, D).
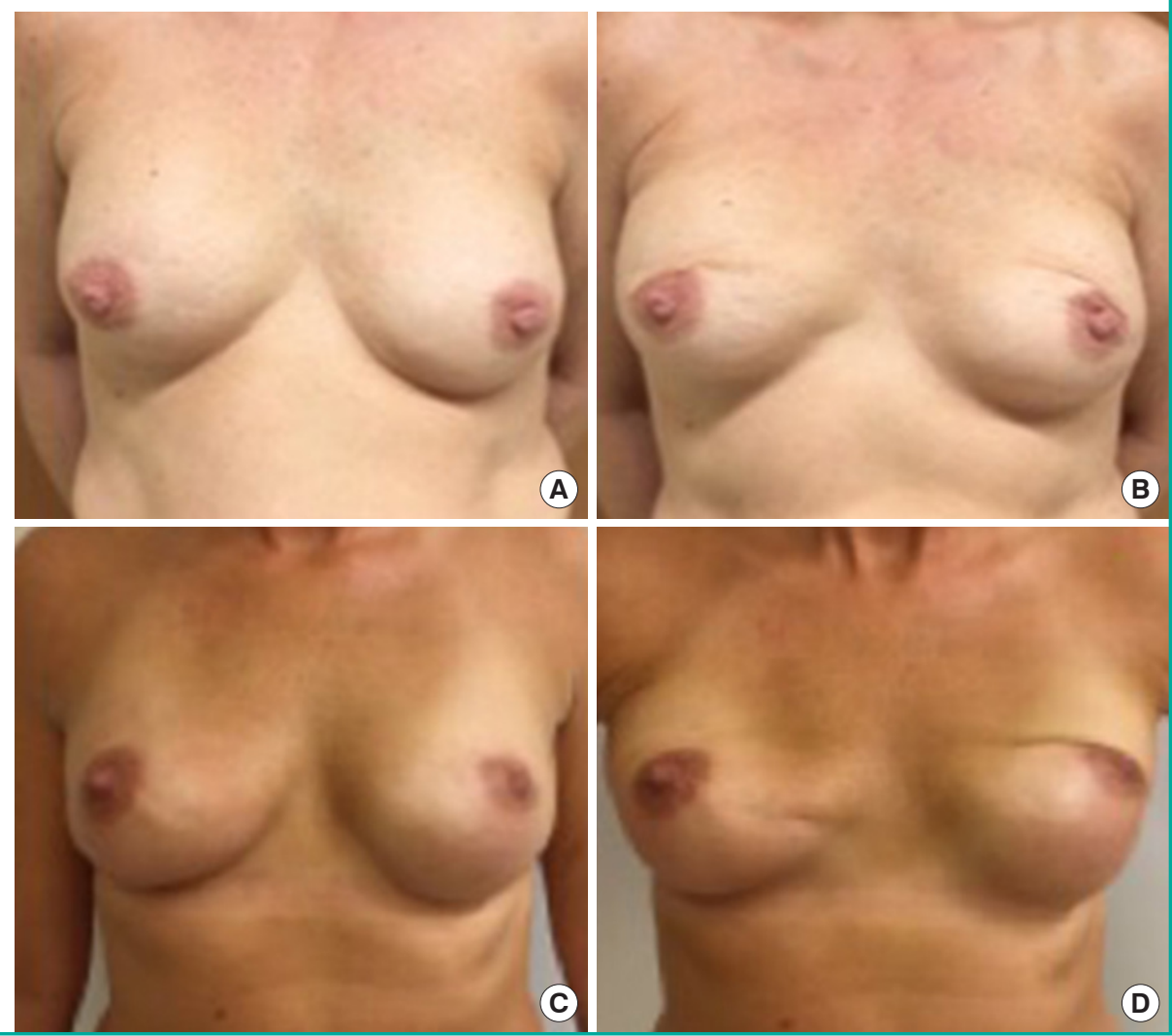

Fig. 4. Breast skin distortion of grades

During activation of the pectoralis major muscle, the images show: moderate (grade 1) distortion of the breast skin ( $A$, $B$ ) and severe (grade 2) distortion of the breast skin $(C, D)$.
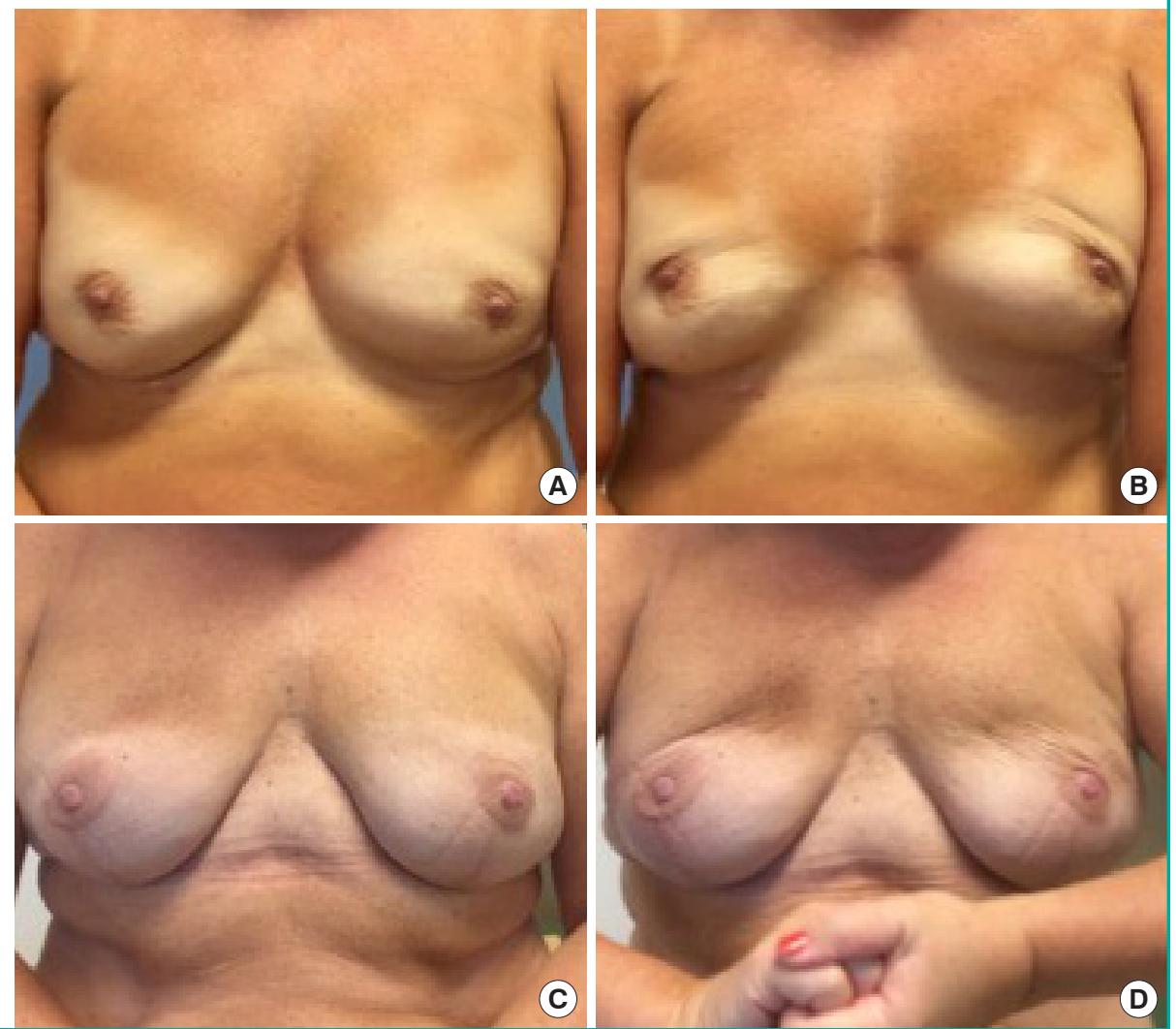


\section{Fig. 5. Movement of the entire breast grades}

During activation of the pectoralis major muscle, the images show: moderate (grade 1) lifting of the entire breast, revealing a visible inframammary crease $\left(A_{\text {, }}\right.$ $B)$ and severe (grade 2) lifting of the entire breast $(C, D)$.
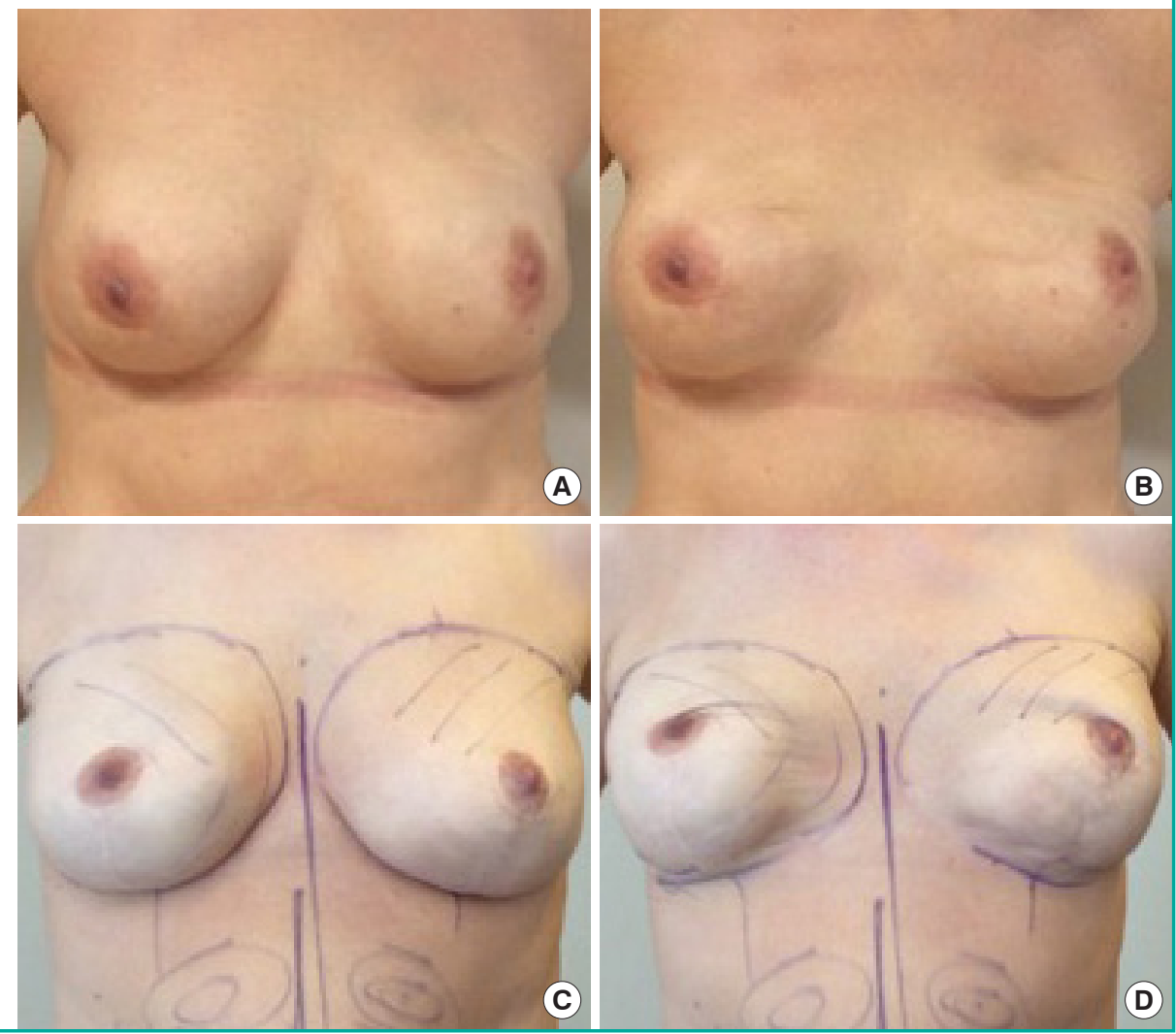

the $\mathrm{TBM} / \mathrm{NAC}$, the skin is darker-colored and the structure is irregular, whereas the smoother and lighter skin surrounding the TBM/NAC was evaluated based on the appearance of skin wrinkles in response to PMM contraction. Each of the three features used in the NSE grading scale was assigned 0 to 2 points depending on the degree of distortion: 0 , no visible distortion/ movement; 1 , visible distortion/movement; or 2 , severe distortion/movement. To distinguish between levels of severity when performing the evaluation, feature 1 (Fig. 3) was classified as moderate when the TBM/NAC moved upwards from its natural position, but remained in a vertical position, while it was classified as severe when the TBM/NAC was horizontalized and kinked inwards. For feature 2 (Fig. 4), evaluation of the breast skin, distortion was classified as moderate when the skin was slightly wrinkled, with wrinkles apparent in only one quadrant, and as severe when the skin was wrinkled with long horizontal lines affecting more than one quadrant. For feature 3 (Fig. 5) movement of the entire breast was classified as moderate when the whole breast was lifted upwards, revealing a visible inframammary crease, and severe when lifted even further. These sub-evaluations were added together to obtain a total score between 0 and 6 points (Table 1). Each video recording was assessed twice by both observers at a 2-month interval.

\section{Table 1. The NSE grading scale}

\begin{tabular}{|l|}
\hline NSE grading scale Right breast Left breast \\
\hline TBM/NAC \\
No distortion: 0 \\
Visible distortion: 1 \\
Severe distortion: 2 \\
Breast skin surrounding TBM/NAC \\
No distortion: 0 \\
Visible distortion: 1 \\
Severe distortion: 2 \\
Entire breast \\
No movement: 0 \\
Visible movement: 1 \\
Severe movement: 2 \\
Total (0-6) \\
\hline NSE, Nipple, Surrounding Skin, Entire Breast; TBM, top of the breast mound; \\
NAC, nipple areolar complex.
\end{tabular}

\section{Statistical analysis}

The Student $t$-test was used to compare the results of the BAD assessments. The null hypothesis was that the degree of $\mathrm{BAD}$ would be equivalent when comparing the subpectoral reconstruction group to the prepectoral reconstruction groups. To further validate our findings, we used kappa statistics to test the 
intra- and inter-rater agreements of the $\mathrm{BAD}$ assessments using our grading system [15]. Agreement between two assessments is purely due to chance when $\kappa=0$, while perfect agreement corresponds to $\kappa=1$. Agreement using kappa statistics is classified as follows: poor, $\kappa<0$, slight, $\kappa=0-0.2$; fair, $\kappa=0.21-0.4$; moderate, $\kappa=0.41-0.60$; substantial, $\kappa=0.61-0.80$; and almost perfect, $\kappa=0.81-1.0[16]$.

\section{RESULTS}

We assessed video recordings of 37 women and 74 breasts. Four women underwent unilateral breast reconstruction (11\%), while 33 underwent bilateral breast reconstruction (89\%). We included 70 reconstructed breasts in the final analysis. Four non-reconstructed breasts were omitted from the analysis. The mean age of the patients was 45 years (standard deviation [SD], 12 years). The mean BMI was $25 \mathrm{~kg} / \mathrm{m}^{2}\left(\mathrm{SD}, 4.3 \mathrm{~kg} / \mathrm{m}^{2}\right)$. There were three smokers $(8 \%)$, and one patient with hypertension (3\%) among the included patients. The mean duration of surgery was 116 minutes (SD, 27 minutes), the mean time until drain removal was 10 days (SD, 4.5 days), and the mean length of hospital stay was 2 days (SD, 1 day). The mean follow-up was 476 days (SD, 391 days). The implant was placed subpectorally in 19 patients (51\%) and prepectorally in 18 patients (49\%) (Table 2).

There was a significant difference in the length of stay between the two groups: $2 \pm 0.57$ days in the prepectoral group compared to $3 \pm 0.96$ days in the subpectoral group $(P=0.001)$. The mean time until drain removal was significantly longer in the subpectoral group (14 days) than in the prepectoral group (7 days) $(\mathrm{P}=0.000)$. The prepectoral reconstruction method tended to be faster, as the mean surgical duration was $15 \mathrm{~min}$ utes shorter than in the subpectoral group $(\mathrm{P}=0.053)$. The follow-up time was significantly different between the two groups defined based on the reconstructive techniques: 272 days in the

Table 2. Demographic characteristics of the prepectoral and subpectoral reconstruction groups

\begin{tabular}{|c|c|c|c|}
\hline Variable & $\begin{array}{l}\text { Prepectoral } \\
(n=18)\end{array}$ & $\begin{array}{c}\text { Subpectoral } \\
(n=19)\end{array}$ & P-value \\
\hline Age (yr) & $44 \pm 9.47$ & $45 \pm 11.97$ & 0.360 \\
\hline BMI $\left(\mathrm{kg} / \mathrm{m}^{2}\right)$ & $24 \pm 3.95$ & $26 \pm 4.62$ & 0.088 \\
\hline Surgical time (min) & $109 \pm 22.32$ & $124 \pm 30.58$ & 0.053 \\
\hline Length of stay (day) & $2 \pm 0.57$ & $3 \pm 0.96$ & 0.001 \\
\hline $\begin{array}{l}\text { Duration of drain } \\
\text { placement (day) }\end{array}$ & $7 \pm 1.64$ & $14 \pm 3.42$ & 0.000 \\
\hline Follow-up (day) & $272 \pm 209$ & $684 \pm 423$ & 0.001 \\
\hline
\end{tabular}

prepectoral group versus 684 days in the subpectoral group $(\mathrm{P}=0.001)$ (Table 2).

We found a significant difference in the degree of $\mathrm{BAD}$ in favor of patients reconstructed by prepectoral implant placement (range, 0.1-0.3) compared to the subpectoral group (range, 3.85.1; $\mathrm{P}=0.000$ ) (Table 3). The inter- and intra-rater agreement was moderate to high. The intra-rater agreement was moderate for surgeon $1(74 \% ; \kappa=0.6)$ and high for surgeon 2 (84\%; $\kappa=0.7$ ), exceeding the expected agreement by $34 \%$ and $37 \%$, respectively. The inter-rater agreement was high ( $88 \%$ at the first assessment and $82 \%$ at the second assessment; $\kappa=0.7-0.8)$, exceeding the expected agreement by $38 \%$ and $35 \%$, respectively (Table 4).

\section{DISCUSSION}

The degree of $\mathrm{BAD}$ following direct-to-implant breast reconstruction was significantly lower in patients with prepectorally placed implants than in those with subpectorally placed implants (Table 3). Furthermore, all (100\%) of the patients who underwent subpectoral direct-to-implant breast reconstruction were assessed to have some degree of $\mathrm{BAD}$, which is higher than

\begin{tabular}{|c|c|c|c|}
\hline & $\begin{array}{l}\text { Prepectoral } \\
(n=34)\end{array}$ & $\begin{array}{l}\text { Subpectoral } \\
\quad(n=36)\end{array}$ & P-value \\
\hline \multicolumn{4}{|l|}{ Surgeon 1} \\
\hline First assessment & $0.2 \pm 0.6$ & $4 \pm 1$ & 0.000 \\
\hline Second assessment & $0.3 \pm 0.6$ & $5.1 \pm 1.1$ & 0.000 \\
\hline \multicolumn{4}{|l|}{ Surgeon 2} \\
\hline First assessment & $0.2 \pm 0.7$ & $3.8 \pm 1.1$ & 0.000 \\
\hline Second assessment & $0.1 \pm 0.4$ & $4.2 \pm 1.2$ & 0.000 \\
\hline
\end{tabular}

Table 4. Intra- and inter-rater agreement using the NSE grading scale

\begin{tabular}{|c|c|c|c|c|}
\hline & $\begin{array}{c}\text { Agreement } \\
(\%)\end{array}$ & $\begin{array}{l}\text { Expected } \\
\text { agreement } \\
(\%)\end{array}$ & $\kappa$-value & $\begin{array}{c}\text { Standard } \\
\text { error }\end{array}$ \\
\hline \multicolumn{5}{|l|}{ Intra-rater } \\
\hline Surgeon $1^{\text {a) }}$ & 74 & 34 & 0.6 & 0.07 \\
\hline Surgeon $2^{\text {a) }}$ & 84 & 37 & 0.7 & 0.08 \\
\hline \multicolumn{5}{|l|}{ Inter-rater } \\
\hline First assessment'b) & 88 & 38 & 0.8 & 0.09 \\
\hline Second assessment ${ }^{\text {b) }}$ & 82 & 35 & 0.7 & 0.08 \\
\hline \multicolumn{5}{|c|}{$\begin{array}{l}\text { NSE, Nipple, Surrounding Skin, Entire Breast. } \\
\text { a'First assessment compared to second assessment; }{ }^{b} \text { Comparison of surgeon } 1 \\
\text { and } 2 \text {. }\end{array}$} \\
\hline
\end{tabular}


the value of $80 \%$ reported by Spear et al. [5] in their series of women who underwent augmentation. The incidence of $\mathrm{BAD}$ in women who underwent reconstruction using the prepectoral technique was very low, which conforms to the results of our recent systematic review, where the incidence of $\mathrm{BAD}$ was found to be close to $0 \%$ following prepectoral implant placement in the included studies [6].

Many studies have stated that the incidence of $\mathrm{BAD}$ was low when prepectoral implant placement was used for direct-to-implant breast reconstruction. However, it has rarely been reported how the degree of $\mathrm{BAD}$ was assessed [6]. In this study, we have clearly defined and classified BAD using our classification system and described how the degree of $\mathrm{BAD}$ was assessed.

We used video recordings for our assessment of BAD. A major advantage of video footage is that dynamic recordings of the breast capture any deformity as it occurs during contraction of the PMM. We find videos to be superior to still photos for evaluating the degree of $\mathrm{BAD}$. Video recordings also facilitate a reproducible evaluation by one or several observers to validate the assessment technique. When observing the videos of patients with direct-to-implant breast reconstructions, we found three areas of the breast that were affected by activation of the PMM: the TBM/NAC, the breast skin surrounding the TBM/NAC, and the movement of the entire breast. The clinical assessment was simple, using three grades, as described in detail above: no visible distortion/movement, visible distortion/movement, and severe distortion/movement. We sought to develop an accurate grading scale for $\mathrm{BAD}$ for women who have undergone reconstruction, which includes the main factors involved in BAD: distortion of the skin and movement of the reconstructed breast. We found that the degree of $\mathrm{BAD}$ was easy to assess using the three parameters of TBM/NAC distortion, breast skin distortion, and breast movement, scoring each from 0 to 2 based on the severity.

We used kappa statistics to test the intra- and inter-rater agreement in the assessments of $\mathrm{BAD}$ [16]. The kappa values in the evaluation of our grading system were high, revealing high consistency in the results (intra-rater agreement, $\kappa=0.6-0.7$; interrater agreement, $\kappa=0.7-0.8$ ). The results revealed a high level of agreement in the assessment of $\mathrm{BAD}$ using our simple clinical NSE grading scale, suggesting that it can be used to assess the degree of $\mathrm{BAD}$ in a reproducible manner.

In comparison to Spear's grading scale, in which the assessment is based on implant visibility, aesthetics, and distortion, we found that the assessment could be simplified by only considering the degree of $\mathrm{BAD} /$ distortion, which seems to be the most important factor leading to an unpleasing aesthetic result, accentuated implant visibility, and functional impairment following direct-to-implant breast reconstruction.

The kappa statistic is well-known as a tool to evaluate the reliability of diagnostic tests $[17,18]$. Its use in plastic surgery is, however, surprisingly scarce. In 2014, Ibrahim et al. [19] used Fleiss' kappa to evaluate aesthetic outcomes following two-stage breast reconstruction with or without $\mathrm{ADM}$, and found kappa values ranging from fair to moderate.

Several methods have been proposed to assess $\mathrm{BAD}[3,7,10$, 20,21]. Most recently, Fracol et al. [21] suggested using Kim's quantitative grading scale to standardize the grading of $\mathrm{BAD}$ by nipple displacement in centimeters and the percentage of skin area affected by rippling [21]. Within the last 2 years, Cheffe et al. [20] developed an objectively measurable scale to address the severity of $\mathrm{BAD}$ in women undergoing dual-plane augmentation. Landmarks on the breast were marked and analyzed on still photos with and without contraction of the PMM. In addition, Kummel et al. [10] proposed a more detailed 3-point grading system to describe and distinguish grades of $\mathrm{BAD}$ in women undergoing augmentation and suggested how to prevent $\mathrm{BAD}$ by performing a two-stage procedure. Furthermore, Vidya and colleagues suggested how to manage $\mathrm{BAD}$ in relation to its grading for women undergoing subpectoral reconstruction [7].

In a systematic literature review, we found that the majority of the proposed grading scales for $\mathrm{BAD}$ had not been tested for reproducibility [6]. If a grading system is to be accessible for general use, it must be tested for reproducibility. The high intra- and inter-rater agreement obtained for our grading system confirms its reproducibility. The NSE grading scale is an easy-to-use, clinically relevant, consistent, and reproducible grading scale for assessing $\mathrm{BAD}$ in women who have undergone reconstruction; therefore, it constitutes a potential contribution to $\mathrm{BAD}$ grading in future clinical studies.

The duration of drain placement was significantly longer in the subpectoral group than in the prepectoral group. A retrospective analysis conducted in 2018 found comparable results between subpectoral and prepectoral reconstructions and no differences in seroma formation [22]. The same conclusion was drawn in a recent large meta-analysis, where the authors found no significant differences in seroma formation between prepectoral and subpectoral reconstructions [23]. In this study, we used one piece of $\mathrm{ADM}$ for both prepectoral and subpectoral reconstruction procedures. Therefore, the difference in seroma formation was not due to the number of pieces of ADM used. Instead, we believe that the difference may have been caused by the more severe surgical trauma that occurred when performing the subpectoral technique.

One of the limitations of this retrospective study is the difference in the duration of follow-up between the subpectoral and 
the prepectoral reconstruction groups. The follow-up in the prepectoral group was short, and the results should be interpreted with this in mind. We cannot rule out the possibility that the degree of $\mathrm{BAD}$ may increase over time in the prepectoral group. Future studies, preferably prospective randomized trials, with longer follow-up will be needed to elucidate this potential issue.

The number of papers on $\mathrm{BAD}$ describing its low incidence following prepectoral direct-to-implant breast reconstructions is increasing. However, although the incidence and degree of $\mathrm{BAD}$ seems to be minimized by using the prepectoral technique, there are also possible disadvantages of using this pocket plane. Specifically, there is a risk of wrinkling, capsular contraction, bottoming out, and visible implant edges. Long-term follow-up of women who undergo prepectoral reconstruction may reveal a substantial incidence of ptosis. Furthermore, this study only examined the degree of $\mathrm{BAD}$ following direct-to-implant breast reconstruction, and no patients reconstructed using a two-stage technique were investigated. The results should be interpreted in this context, and we cannot know whether the results are applicable for patients reconstructed using a two-stage technique.

Most importantly, we must keep in mind patients' perceptions of BAD. Studies evaluating and comparing cosmetic and functional outcomes, as well as patient-related outcome measures, following prepectoral and subpectoral direct-to-implant breast reconstruction are needed. Surgeons may desire a shift towards the prepectoral technique, but is this wish also shared by patients?

The degree of $\mathrm{BAD}$ following direct-to-implant breast reconstruction was significantly lower in patients with prepectorally placed implants than in those with subpectorally placed implants. We found some degree of $\mathrm{BAD}$ in all cases of subpectoral direct-to-implant breast reconstruction. $\mathrm{BAD}$ can be assessed in a reproducible manner using the NSE grading scale. In conclusion, we have developed a supplementary grading scale that we believe is easy-to-use, clinically relevant, consistent, and reproducible for the assessment of $\mathrm{BAD}$.

\section{NOTES}

\section{Conflict of interest}

No potential conflict of interest relevant to this article was reported.

\section{Ethical approval}

The study was approved by the Regional Committee on Health Research Ethics for Southern Denmark (S-20160160) and performed in accordance with the principles of the Declaration of Helsinki. Written informed consents were obtained.

\section{Patient consent}

The patients provided written informed consent for the publication and the use of their images.

\section{Author contribution}

Writing original draft: Dyrberg DL, Thomsen JB. Methodology: Thomsen JB, Bille C. Formal analysis: Dyrberg DL, Thomsen JB. Project administration: Thomsen JB, Sørensen JA. Data curation: Gunnarsson GL. Writing- review and editing: Dyrberg DL, Thomsen JB, Bille C, Sørensen JA, Gunnarsson GL. Visualization: Dyrberg DL, Thomsen JB.

\section{ORCID}

Diana Lydia Dyrberg https://orcid.org/0000-0002-1537-7703

Gudjon Leifur Gunnarsson https://orcid.org/0000-0001-73889451

Camilla Bille https://orcid.org/0000-0002-7727-462X

Jens Ahm Sørensen https://orcid.org/0000-0003-4903-0094

Jørn Bo Thomsen https://orcid.org/0000-0002-7368-6133

\section{REFERENCES}

1. Pelle-Ceravolo M, Del Vescovo A, Bertozzi E, et al. A technique to decrease breast shape deformity during muscle contraction in submuscular augmentation mammaplasty. Aesthetic Plast Surg 2004;28:288-94.

2. Strasser EJ. Results of subglandular versus subpectoral augmentation over time: one surgeon's observations. Aesthet Surg J 2006;26:45-50.

3. Becker H, Fregosi N. The impact of animation deformity on quality of life in post-mastectomy reconstruction patients. Aesthet Surg J 2017;37:531-6.

4. Gabriel A, Sigalove S, Sigalove NM, et al. Prepectoral revision breast reconstruction for treatment of implant-associated animation deformity: a review of 102 reconstructions. Aesthet Surg J 2018;38:519-26.

5. Spear SL, Schwartz J, Dayan JH, et al. Outcome assessment of breast distortion following submuscular breast augmentation. Aesthetic Plast Surg 2009;33:44-8.

6. Dyrberg DL, Bille C, Gunnarsson GL, et al. Breast animation deformity. Arch Plast Surg 2019;46:7-15.

7. Vidya R, Tafazal H, Salem F, et al. Management based on grading of animation deformity following implant-based subpectoral breast reconstruction. Arch Plast Surg 2018;45: 185-90.

8. Bertozzi N, Pesce M, Santi P, et al. One-stage immediate breast reconstruction: a concise review. Biomed Res Int 2017; 


\section{7:6486859.}

9. Gunnarsson GL, Thomsen JB. Prepectoral hammock and direct-to-implant breast reconstruction in 10 minutes: a focus on technique. Plast Reconstr Surg Glob Open 2018;6:e1931.

10. Kummel S, Kummel A, Hagemann F, et al. Jumping breast phenomenon following subcutaneous mastectomy: first description and grading of a well-known breast deformity. Breast Care (Basel) 2018;13:354-8.

11. Egeberg A, Sorensen JA. The impact of breast implant location on the risk of capsular contraction. Ann Plast Surg 2016; 77:255-9.

12. Gunnarsson GL, Borsen-Koch M, Wamberg P, et al. How to perform a NAC sparing mastectomy using an $\mathrm{ADM}$ and an implant. Gland Surg 2014;3:252-7.

13. Dyrberg DL, Bille C, Gunnarsson GL, et al. Visualized preand subpectoral implant placement for immediate breast reconstruction. Gland Surg 2019;8(Suppl 4):S251-4.

14. Random.org. True random number service [Internet]. Dublin, Randomness and Integrity Services Ltd. [cited 2019 Feb 21]. Available from: https://www.random.org/.

15. Cohen J. A coefficient of agreement for nominal scales. Educ Psychol Meas 1960;20:37-46.

16. Landis JR, Koch GG. The measurement of observer agreement for categorical data. Biometrics 1977;33:159-74.
17. Elmore JG, Wells CK, Lee CH, et al. Variability in radiologists' interpretations of mammograms. N Engl J Med 1994; 331:1493-9.

18. Fleiss JL. Measuring nominal scale agreement among many raters. Psychol Bull 1971;76:378-82.

19. Ibrahim AM, Koolen PG, Ganor O, et al. Does acellular dermal matrix really improve aesthetic outcome in tissue expander/implant-based breast reconstruction? Aesthetic Plast Surg 2015;39:359-68.

20. Cheffe MR, Valentini JD, Collares MV, et al. Quantifying dynamic deformity after dual plane breast augmentation. Aesthetic Plast Surg 2018;42:716-24.

21. Fracol M, Feld LN, Chiu WK, et al. An overview of animation deformity in prosthetic breast reconstruction. Gland Surg 2019;8:95-101.

22. Chandarana MN, Jafferbhoy S, Marla S, et al. Acellular dermal matrix in implant-based immediate breast reconstructions: a comparison of prepectoral and subpectoral approach. Gland Surg 2018;7(Suppl 1):S64-9.

23. Chatterjee A, Nahabedian MY, Gabriel A, et al. Early assessment of post-surgical outcomes with pre-pectoral breast reconstruction: a literature review and meta-analysis. J Surg Oncol 2018;117:1119-30. 